\section{OC-013 HOW OFTEN IS UPPER GASTROINTESTINAL CANCER MISSED DURING ENDOSCOPY?}

doi:10.1136/gutjnl-2013-304907.013

1."D Cheung, ${ }^{2} \mathrm{~T}$ Evans, ${ }^{2} \mathrm{G}$ Lawrence, ${ }^{1} \mathrm{~N}$ Trudgill. 'Department of Gastroenterology, Sandwell General Hospital, West Bromwich; ${ }^{2}$ West Midlands Cancer Intelligence Unit, Birmingham, UK

Introduction Meta-analysis of published literature suggests that up to $14 \%$ of upper gastrointestinal cancer (UGIC) subjects had a negative oesophagogastroduodenoscopy (OGD) up to 3 years prior to diagnosis. We have examined how often UGIC is missed at OGD in our organisation and associated risk factors.

Methods Computerised OGD records from Sandwell General and City Hospitals between 1999 and 2007 were retrieved and submitted to the West Midlands Cancer Intelligence Unit (WMCIU) for UGIC registrations linkage. Subjects undergoing OGD 3 months to 5 years before diagnosis were identified as potentially missed UGIC cases and those with no OGD 3 months to 5 years before diagnosis served as controls. The influence of age, gender, indication, endoscopist specialty, trainee involvement, sedation use, number of biopsies taken, site and histology of UGIC on missed UGIC were examined by logistic regression analysis.

Results 36577 OGD records were submitted to WMCIU for cancer linkage and 524 UGIC were linked. 419 control UGIC: 388 (93\%) diagnosed at initial OGD; 31 (7\%) diagnosed at repeat OGD appropriately (within 90 days, with previous benign histology). 105 (20\%) potentially missed UGIC cases: 39 (37\%) oesophageal cancer (OC) and $66(63 \%)$ gastric cancer (GC); $42(40 \%)$ had OGD 3 months to 1 year prior to diagnosis; 33 (31\%) had OGD 1-3 years prior and 30 (29\%) had OGD 3-5 years prior. Furthermore, 30\% of missed UGIC cases had OGD where abnormality at site of UGIC was seen but not biopsied or benign histology from an inadequate number of biopsies $(<4)$ within 3 months to 1 year prior to diagnosis.

Lack of alarm symptoms $(2.51,95 \%$ CI $1.58-4.00, p=0.0001)$ and female gender $(1.79,1.16-2.79, p=0.009)$ were associated with missed UGIC. The number of biopsies taken was significantly lower in the missed UGIC group than in the controls $(2.1 \pm 0.2$ vs $5.4 \pm 0.1, p<0.05)$

In subjects with $\mathrm{OC}$, mid-oesophageal $\mathrm{OC}$ appeared more likely to be missed than lower-oesophageal OC $(2.04,0.99-4.23, p=0.05)$. Oesophageal squamous cell carcinoma was much more likely to be missed than oesophageal adenocarcinoma (4.47, 1.88-10.65, $p<0.001)$. In GC subjects, there was no association between missed UGIC and tumour site $(1.10,0.61-1.97, p=0.3)$ or histology subtype $(1.10,0.46-2.67, p=0.8)$.

Age (1.5, -0.8-3.8, p=0.2), endoscopist specialty $(1.39,0.70-2.76$, $\mathrm{p}=0.34)$, trainee involvement $(1.2,0.78-1.86, \mathrm{p}=0.39)$ and sedation use $(0.98,0.64-1.51, p=0.9)$ were not associated with increased risk of missing UGIC.

Conclusion Missing UGIC at OGD was seen in 14.3\% of subjects within 3 years of diagnosis. It was associated with lack of alarm symptoms, female gender, oesophageal squamous cell carcinoma and an insufficient number of biopsies from recognised abnormalities.

Disclosure of Interest None Declared

\section{IBD free papers}

\section{OC-014 CLINICAL AND ENDOSCOPIC IMPROVEMENT FOLLOWING HEMOPOIETIC STEM CELL TRANSPLANTATION VS MOBILISATIION ALONE IN CROHN'S DISEASE}

doi:10.1136/gutjnl-2013-304907.014

1,"C Hawkey, ${ }^{2} \mathrm{M}$ Allez, ${ }^{3} \mathrm{~S}$ Ardizzone, ${ }^{4} \mathrm{~L}$ Clark, ${ }^{5} \mathrm{~J}-\mathrm{F}$ Columbel, ${ }^{6} \mathrm{~S}$ Danese, ${ }^{7} \mathrm{D}$ Farge-Bancel, ${ }^{8} \mathrm{M}$ Labopin, ${ }^{9} \mathrm{~J}$ Lindsay, ${ }^{1} \mathrm{~A}$ Norman, ${ }^{10} \mathrm{~F}$ Onida, ${ }^{11} \mathrm{E}$ Ricart, ${ }^{12} \mathrm{G}$ Rogler, ${ }^{13} \mathrm{M}$ Rovira, ${ }^{1} \mathrm{~N}$ Russell, ${ }^{14} \mathrm{~J}$ Satsangi, ${ }^{15} \mathrm{~S}$ Travis, ${ }^{16} \mathrm{~A}$ Tyndall, ${ }^{17} \mathrm{~S}$ Vermeire. ${ }^{1}$ Nottingham Digestive
Diseases Centre, University of Nottingham, Nottingham, UK; ${ }^{2}$ Service de Gastroentérologie, Hospital Saint-Louis, Paris, France; ${ }^{3}$ IBD Unit, L Sacco University Hospital, Milan, Italy; ${ }^{4}$ EBMT Clinical Trials, European Group for Blood \& Marrow Transplantation, London, UK; ${ }^{5} \mathrm{CHRU}$ Lille, Hospital Huriez, Lille, France; ${ }^{6}$ Dept of Gastroenterology, Instituto Clinico Humanitas, Milan, Italy; 'Unite de Medecine Interne et Pathologie Vasculaire, Hospital Saint-Louis; ${ }^{8}$ European Group for Blood \& Marrow Transplantation, Paris, France: ${ }^{9}$ Digestive Diseases CAU, Barts \& The London School of Medicine, London, UK; ${ }^{10}$ Hematology-BMT Center, University of Milan, Milan, Italy; "Gastroenterology Department-CIBER-EHD, Hospital Clinic, Barcelona, Spain; ${ }^{12}$ Klinik für Gastroenterologie und Hepatologie, University Hospital of Zurich, Zurich, Switzerland; ${ }^{13} B M T$ Unit, Hospital Clinic, Barcelona, Spain; ${ }^{14}$ Gastrointestinal Unit, Molecular Medicine Unit, University of Edinburgh, Edinburgh; ${ }^{15}$ Translational Gastroenterology Unit, John Radcliffe Hospital, Oxford, UK; ${ }^{16}$ Dept of Rheumatology, University Hospital Basel, Basel, Switzerland; ${ }^{17}$ Department of Gastroenterology, University Hospitals Leuven, Leuven, Belgium

Introduction The Autologous Stem Cell Transplantation International Crohn's Disease (ASTIC) Trial is a randomised controlled trial co-sponsored by ECCO and EBMT and funded by the Broad Foundation that investigates immunoablation and hemopoietic stem cell transplantation (HSCT) in Crohn's disease (CD) over 1 year: all patients will have reached this endpoint by April 2013.

Methods Patients with impaired quality of life due to active CD, despite $\geq 3$ immunosuppressive agents all underwent mobilisation (iv cyclophosphamide $4 \mathrm{gm} / \mathrm{M}^{2}$ over 2 days then filgrastim $10 \mu / \mathrm{kg} /$ day) before randomisation to immediate (1 month) or delayed (13 months) HSCT. The conditioning regime was iv cyclophosphamide $50 \mathrm{mg} / \mathrm{kg}$ per day for 4 days, anti-thymocyte globulin $2.5 \mathrm{mg} / \mathrm{kg} /$ day and methyl prednisolone $1 \mathrm{mg} / \mathrm{kg}$ on days $3-5$. The bone marrow was reconstituted by infusion of an unselected graft of $3-8 \times 10^{6} / \mathrm{kg}$ CD34 +ve stem cells. Clinical (CDAI), endoscopic (SES-CD) quality of life and safety data are compared 1 year after mobilisation alone or after mobilisation and HSCT.

Results As of Jan 2013, data are available on 34/45 patients. Following mobilisation and HSCT, the CDAI fell from 317 (median, IOR 244-407) to $157(71-246, \mathrm{n}=17)$ vs 351 (313-446) and 298 $(220-370, n=17)$ with mobilisation alone. The aggregate lower GI SES-CD score was 13.0 (8.5-24.5) before and 3.0 (1.5-10.0) after HSCT compared to 13.0 (6.5-15.5) before and 6.5 (3.5-17.8) after mobilisation alone. Over the whole study to end 2012 there were 62 SAEs in 19 patients randomised to early transplantation (3.3 per patient) and 58 in 18 patients randomised to delayed transplantation (3.2 per patient). One patient died following HSCT. Final results of the study will be available to be presented for the first time at BSG 2013

Conclusion Immunoablation and HSCT appears to be an effective treatment for CD that may substantially reduce endoscopic evidence of disease but incurs significant toxicity. The final results of the trial will allow a rational evaluation of the effectiveness and safety of HSCT to be discussed at BSG 2013.

Disclosure of Interest None Declared

\section{OC-015 THE INFLUENCE OF GENDER AND HAEMOGLOBIN ON TPMT ACTIVITY}

doi:10.1136/gutjnl-2013-304907.015

1." P A Blaker, 'V C Kariyawasam, 'K V Patel, 'R M Goel, 'M G Ward, 'P M Irving, ${ }^{2}$ A M Marinaki, ' J D Sanderson. 'Gastroenterology; ${ }^{2}$ Purine Research Laboratory, Guys and St Thomas Hospitals' NHS Foundation Trust, London, UK

Introduction Pre-treatment measurement of red blood cell (RBC) thiopurine-S-methyltransferase (TPMT) activity is recommended to guide initial dosing of azathioprine (AZA) and mercaptopurine (MP). TPMT exhibits a trimodal distribution, with low and intermediate activities predicting myelotoxicity at standard drug doses. There is a high concordance between TPMT genotype and normal or low enzyme activity (93-100\%); however the relationship is poor in the intermediate range (53-100\%) ${ }^{[1]}$. Furthermore, there 